\title{
Artículos
}

\section{Comercio electrónico: la fidelización del usuario}

\author{
Por Pablo Lara Navarra y José Ángel Martínez Usero
}

\begin{abstract}
Resumen: Se destaca la situación actual de redimensionamiento de internet en el ámbito de los negocios digitales, a través del estudio del concepto de comercio electrónico, junto a las bases para la puesta en funcionamiento de un proyecto de dicha actividad. Se justifica la importancia de las herramientas de fidelización en el éxito de los proyectos de comercio electrónico y se propone un modelo que representa la función del sistema de información organizacional en los procesos de captación del cliente. Se concluye destacando la necesidad de integrar las herramientas de tratamiento de la información sobre los clientes con el sistema de información global. Asimismo, el feedback originado por el sistema de información debe integrarse en los procesos de negocio con el objetivo de mejorar los servicios y la satisfacción del cliente.
\end{abstract}

Palabras clave: Comercio electrónico, Fidelización, CRM, VRM, Sistema de información, Marketing, Estrategia de negocio.

\section{Title: E-commerce: building customer loyalty}

Abstract: The article discusses the current reshaping of internet as regards digital business. Definitions are provided for the concept of electronic commerce and the foundations on which to develop an e-commerce project are described. High-

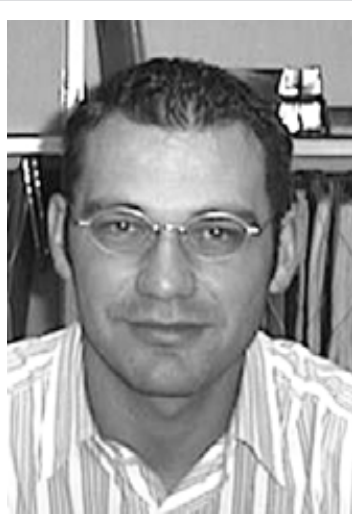

José Ángel Martínez Usero. Diplomado en biblioteconomía y documentación, licen ciado en documentación y máster en sistemas de información. Ha trabajado como documentalista en diferentes organizaciones privadas y como website manager para Calderdale MBC (UK). Es consultor de la licenciatura en documentación de la UOC y profesor asociado de la Universidad Carlos III. Actualmente desarrolla proyectos relacionados con la gestión del conocimiento y la administración electrónica. lighted is the importance of tools for building customer loyalty for successful e-commerce projects and a model is proposed for representing the relationship between the corporate information system and processes for capturing clients. Finally, the authors stress the need to integrate the tools managing client information with the overall corporate information system. Likewise, the feedback generated by the information system must be incorporated back into business procedures in order to improve client services and satisfaction.

Keywords: Electronic commerce, Loyalty, Customisation, CRM, VRM, Information system, Marketing, Business strategy.

Lara Navarra, Pablo; Martínez Usero, José Ángel. «Comercio electrónico: la fidelización del usuario». En: El profesional de la información, 2002, noviembre-diciembre, v. 11, n. 6, pp. 408-420.

\section{Introducción}

«El comercio electrónico es para la revolución de la información lo que fue el ferrocarril para la revolución industrial: un desarrollo totalmente nuevo e inesperado. Y así como la revolución industrial abrió un abismo con el pasado, el ferrocarril cambió para siempre la concepción mental de las distancias; la revolución de la información, de la mano del comercio electrónico, alterará las esferas políticas, económicas y sociales». Peter Drucker en: Gestión, 2000, enero.
Algunos expertos apuntan el inicio de un punto de inflexión o deceleración de los modelos e-commerce , e-business o cualquier modelo de organización que tenga antepuesta la famosa "e". En el informe de 2001 de la Conferencia sobre comercio y desarrollo de las Naciones Unidas se indica que el descenso y desaparición de empresas puntocom, junto a las caídas bursátiles, responden a muchas de las pautas de anteriores revoluciones tecnológicas. Se llega a comparar incluso con el inicio de la industria automovilística y cómo la

Artículo recibido el 19-09-02

Aceptación definitiva: 25-10-02 


\section{Filosofía just in time (jit) y el comercio electrónico}

Se basa en el principio de la producción ajustada y es definida como un conjunto de principios y técnicas operativas que intentan ajustar los procesos empresariales a las demandas del cliente. Pretende eliminar la sobreproducción o stocks en la producción y generar a partir de la detección de las demandas. Esta filosofía conlleva un fuerte control en la gestión de información, pues un error en la misma puede producir una parada en la producción, así como la no entrega a tiempo del pedido.

Este modelo de trabajo afecta a la cadena completa de producción desde el diseño hasta la venta. Los sistemas jit reducen el tiempo de ejecución del producto y permiten responder con mayor flexibilidad al mercado.

Introducir esta filosofía bajo un modelo de comercio electrónico equivale a la automatización de casi la totalidad de las tareas de la cadena de producción, que previamente han sido parametrizadas a través de los sistemas de información de la organización, dando por sentado que la parametrización y automatización se realizan a partir del conocimiento del mercado sobre el que se actúa, junto al organigrama de la cadena de producción y los clientes finales. Este conocimiento permite producir según las demandas y aumentar la bolsa de productos dependiendo de las variables de mercado detectadas. La falta de control del workflow y del retorno de información en la gestión puede desencadenar errores irreparables.

desaparición de muchos de los primeros fabricantes no ha repercutido en la importancia de la industria del transporte en la economía actual. Ante estos datos podríamos indicar, más acertadamente, que nos encontramos en un momento de redimensionamiento de la internet comercial y de los servicios ofertados a la comunidad red.

Tras una época de multimillonarias inversiones en proyectos poco sólidos, y superada la panacea de internet como medio enriquecedor a corto plazo, se está realizando un primer reajuste de los modelos de co- mercio electrónico implantados durante este estadio que todavía no ha concluido. Se está produciendo un reajuste en los servicios y productos de un mercado que aparenta carencias de adaptación a la sociedad de la información o, como algunas empresas han señalado en el cierre de sus proyectos de comercio electrónico, un mercado inmaduro para las apuestas tecnológicas desarrolladas. Pero estas noticias desalentadoras hacen referencia al crecimiento global de internet, no a los datos de comercio como aparecen en los diferentes estudios y análisis del mercado electrónico. Éstos

\section{Legislación más relevante sobre comercio electrónico en España}

\section{Comercio electrónico.}

-Sociedad de la información, corrección de error de la Ley 34/2002, de II de julio, de servicios de la sociedad de la información y de comercio electrónico.

—Sociedad de la información. Ley 34/2002, de II de julio, de servicios de la sociedad de la información y de comercio electrónico.

\section{Firma electrónica.}

-Firma electrónica. Real Decreto-ley 14/1999, de 17 de septiembre, sobre firma electrónica.

-Firma electrónica. Resolución de 21 de octubre de 1999, del Congreso de los Diputados, por la que se ordena la publicación del acuerdo de convalidación del Real Decreto-ley 14/1999, de 17 de septiembre, sobre firma electrónica.

-Firma electrónica. Orden de 21 de febrero de 2000 por la que se aprueba el reglamento de acreditación de prestadores de servicios de certificación y de certificación de determinados productos de firma electrónica.

-Firma electrónica. Orden de 21 de febrero de 2000 por la que se aprueba el reglamento de acreditación de prestadores de servicios de certificación y de certificación de determinados productos de firma electrónica.

-Notarios y registradores de la propiedad y mercantiles. Instrucción de 19 de octubre de 2000 , de la Dirección General de los Registros y del Notario, sobre el uso de la firma electrónica de los fedatarios públicos.

-Firma electrónica. Ley $3 / 2002$, de 21 de mayo, para el desarrollo del uso de la firma electrónica en las administraciones públicas de la Comunidad Autónoma de la Rioja. 


\begin{tabular}{|c|c|c|c|c|c|c|c|c|}
\hline \multicolumn{9}{|c|}{ Evolución del comercio electrónico a través de medios de pago en España. Interanual 2001-2002 } \\
\hline & \multicolumn{2}{|c|}{ Julio-septiembre 2001} & \multicolumn{2}{|c|}{ Octubre-diciembre 2001} & \multicolumn{2}{|c|}{ Enero-marzo 2002} & \multicolumn{2}{|c|}{ Abril-junio 2002} \\
\hline $\begin{array}{l}\text { Tipo } \\
\text { transacción }\end{array}$ & $\begin{array}{l}\mathrm{N}^{0} \text { de } \\
\text { operaciones }\end{array}$ & Miles de $€$ & $\begin{array}{l}\text { No de } \\
\text { operaciones }\end{array}$ & Miles de $€$ & $\begin{array}{l}\mathrm{N}^{\circ} \text { de } \\
\text { operaciones }\end{array}$ & Miles de $€$ & $\begin{array}{l}N^{\circ} \text { de } \\
\text { operaciones }\end{array}$ & Miles de $€$ \\
\hline $\begin{array}{l}\text { Transacciones } \\
\text { españolas en el } \\
\text { exterior }\end{array}$ & 239.084 & 13.688 & 319.897 & 19.686 & 378.609 & 23.673 & 438.169 & 27.130 \\
\hline $\begin{array}{l}\text { Transacciones } \\
\text { del exterior en } \\
\text { España }\end{array}$ & 53.559 & 5.278 & 58.086 & 7.050 & 73.776 & 8.732 & 85.224 & 9.901 \\
\hline $\begin{array}{l}\text { Transacciones } \\
\text { en España }\end{array}$ & 274.455 & 9.736 & 430.027 & 12.989 & 405.837 & 15.149 & 337.647 & 16.837 \\
\hline Total & 567.098 & 28.702 & 808.010 & 39.725 & 858.222 & 47.554 & 861.040 & 53.867 \\
\hline
\end{tabular}

Tabla 1. Evolución del comercio electrónico en España. Fuente: Comisión de Mercado de las Telecomunicaciones.

indican y apuestan por el comercio electrónico como motor de crecimiento e influencia en la economía actual, como podemos comprobar en el estudio de la Asociación Española de Comercio Electrónico con la colaboración del Ministerio de Ciencia y Tecnología (figura 1) y en el $8^{\circ}$ informe sobre comercio electrónico en España a través de entidades de medios de pago (tabla 1).

\section{Qué es el comercio electrónico}

El término comercio electrónico ha obtenido últimamente una mayor divulgación, aunque ha existido bajo diversas formas desde hace más de 20 años. Las tecnologías EDI (electronic data interchange) y EFT (electronic fund transfer) aparecieron a finales de los años 70. De igual forma, los servicios bancarios por teléfono son otra modalidad de comercio electrónico que comenzó a principios de los 80 en EUA. A mediados de la década se extendieron tecnologías de apoyo al comercio electrónico totalmente diferentes que proporcionan servicios online y una nueva forma de interacción social, tales como news groups, file transfer protocol (ftp) e inter-relay chat (irc).

En los 90, la aparición de la world wide web en internet representa una solución tecnológica fácil de usar

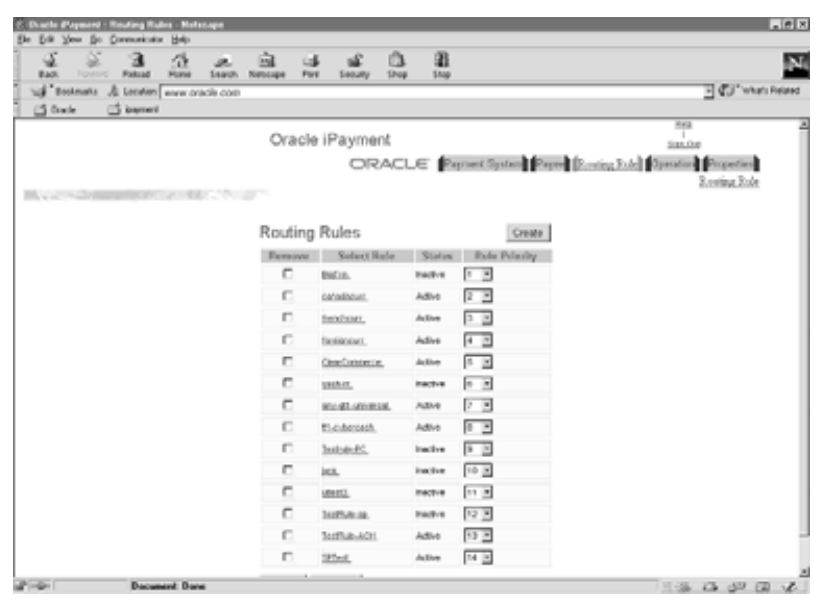

para el problema de la publicación y diseminación de la información. El web ofrece al comercio electrónico una vía más barata de hacer negocios y permite actividades empresariales diversas.

\section{«El comercio electrónico no na- ce con internet, sino que ésta es utilizada como plataforma para su despegue y desarrollo»}

Comercio electrónico es definido por los expertos como la utilización de la informática y las telecomunicaciones para canalizar los flujos de información y operaciones de negocio existentes entre una empresa y sus interlocutores de negocio (clientes, proveedores, entidades financieras, etc.). Esta definición permite deducir que engloba cualquier transacción no convencional entre dos o más actores a través de medios electrónicos.

Según el proyecto piloto del G7, se trata de un concepto general que abarca cualquier tipo de interacción comercial realizada usando la tecnología de la información y las telecomunicaciones (TIC). Incluye el intercambio electrónico de bienes (mercancías o servicios) tangibles o intangibles, así como la publicidad y

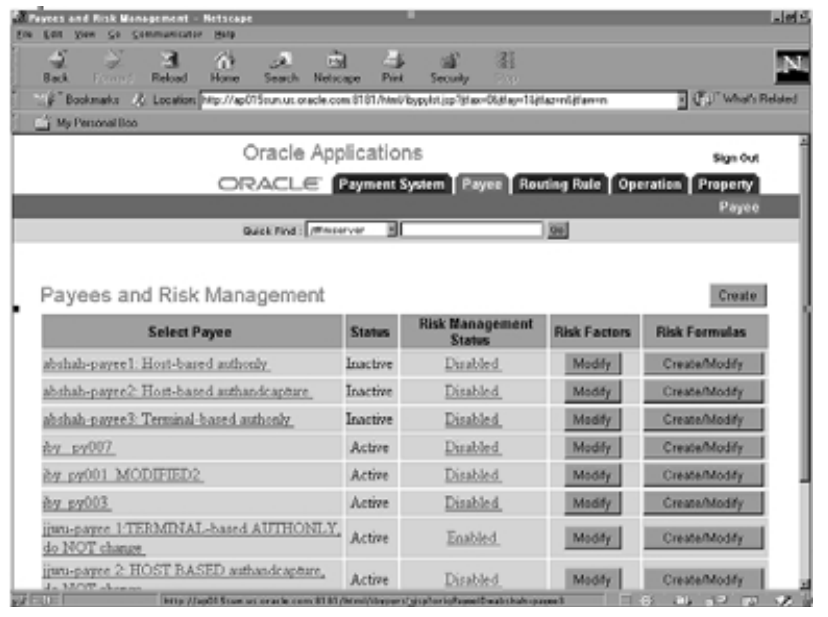


promoción de productos y servicios, el intercambio de contactos entre comerciales, servicios de apoyo postventa, etc. ${ }^{1}$.

La Unión Europea, en la comunicación de la Comisión de 18 de abril de 1997, define el comercio electrónico como un modelo basado en el tratamiento electrónico y de transmisión de datos, que abarca actividades muy diversas que van desde el intercambio de bienes y servicios a la entrega en línea de información digital, pasando por la transferencia electrónica de fondos, la actividad bursátil, la contratación pública, etc. No se limita a internet, sino que incluye otras aplicacio-
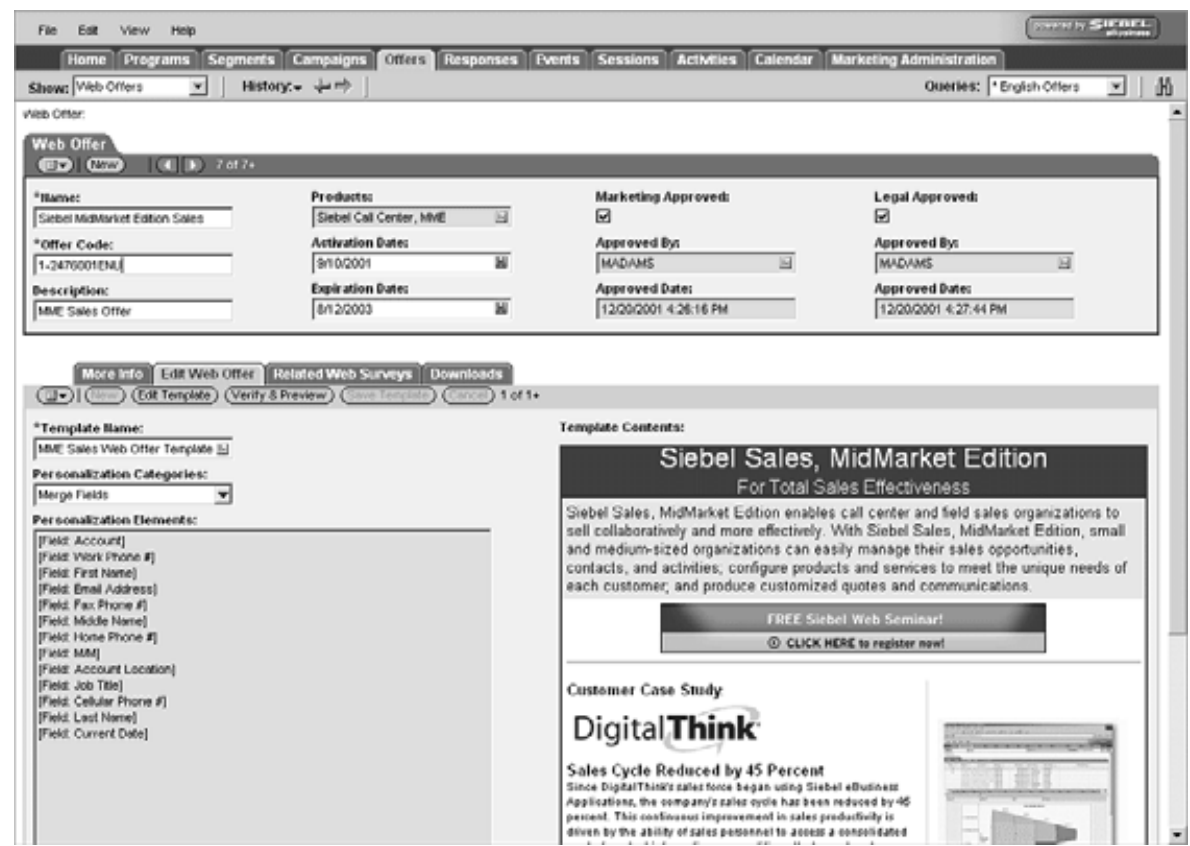
nes tales como el videotexto, la telecompra, la compra por catálogo o por cd-rom²

Las actividades son clasificadas en 2 categorías:

-El comercio-e indirecto: pedido electrónico de bienes tangibles con entrega física.

-El comercio-e directo: pedido en línea, el pago y la entrega de bienes y servicios intangibles.

El comercio electrónico, junto a las aplicaciones ebusiness, comprende todos los procesos con los diferentes actores de la actividad empresarial y comercial (distribuidores, proveedores, clientes, socios, empleados, etc.), incluyendo acciones como ventas, marketing, registro, entregas, pagos, captación, fidelización, etc. Ello implica la utilización de aplicaciones muy diversas, entre otras: TPV (terminales punto de venta, cajeros, generalmente conectados a una red; en este artículo nos referimos a los TPV virtuales, a través de internet), servicios de sindicación (venta de información preseleccionada), servicios a medida, aplicaciones EDI (electronic data interchange), sistemas ERP (en-

terprise resource planning), CRM (customer relationship management), gestión de información y sistemas colaborativos (tabla 2).

\section{Clasificación del comercio electrónico.}

Puede subdividirse en 4 grandes categorías:

-Empresa-empresa: entre compañías. Business to business (B2B).

-Empresa-consumidor: entre empresas y consumidores. Business to consumer (B2C).

-Empresa-administración: entre empresa y la administración. Business to administration (B2A).

-Consumidor-administración: entre consumidores y la administración. Consumer to administration (C2A).

\section{Acciones para el desarrollo de un proyecto de comercio electrónico}

No existe un único modelo de éxito, ya que el comercio electrónico se encuentra en una fase de redimensionamiento en espera de los datos de crecimiento

\begin{tabular}{|l|l|}
\hline Comercio electrónico tradicional & Comercio electrónico en internet \\
\hline Sólo entre empresas & $\begin{array}{l}\text { Empresas-consumidores } \\
\text { Empresas-empresas } \\
\text { Empresas-administración } \\
\text { Consumidor-administración }\end{array}$ \\
\hline Círculos cerrados, a menudo específicos de un sector & Mercado mundial abierto \\
\hline Número limitado de participantes empresariales & Número ilimitado de participantes \\
\hline Redes cerradas propias & Redes abiertas, no protegidas \\
\hline Participantes conocidos y dignos de confianza & Participantes conocidos y desconocidos \\
\hline La seguridad forma parte del diseño de la red & Son necesarias seguridad y autentificación \\
\hline El mercado es un círculo & La Red es el mercado \\
\hline
\end{tabular}
de internet, junto a los primeros resultados de las políticas gubernamentales y programas privados de comercio electrónico en la sociedad de la información. Pero sí podemos apuntar unos criterios o factores a tener en cuenta.

\section{Establecer una estrategia or- ganizacional.}

Un proyecto de estas características debe determinar con claridad la estrategia de negocio y las téc- 
nicas necesarias para su implementación. Además, se ha de establecer una planificación pormenorizada de la puesta en marcha del servicio en cuanto a objetivos, logística, costes, beneficios, riesgos, personal y tecnología.

«El comercio electrónico, junto a las nuevas técnicas y modelos de organización, tiende hacia la gestión integral de las organizaciones posibilitando unir todos los procesos bajo un único sistema de información que ayude a la toma de decisiones estratégicas"

Un plan de negocio es el documento en el que la organización sintetiza su estrategia de negocio. En él se recogen todos los aspectos del proyecto de un modo ordenado y sistemático, con el fin de presentarlo para su aprobación al equipo directivo, para interesar a posibles inversionistas, conseguir créditos, ayudas y subvenciones, etc. Los principales elementos que ha de incluir un plan de negocio son los siguientes:

-Resumen ejecutivo (síntesis del documento con las principales conclusiones y recomendaciones)

-Misión del servicio, concreción de la razón de ser del proyecto.

-Análisis dafo (de las debilidades, amenazas, fortalezas y oportunidades).

-Objetivos estratégicos.

- Recursos humanos. Personas que intervienen, funciones y tareas dentro de las diferentes fases del proyecto.

—Evaluación de costes.

-Plazos de realización, donde se incluyen las fechas de las diferentes fases de desarrollo.

-Método de seguimiento y evaluación.

Un rasgo a valorar en su viabilidad es la capacidad de establecer estrategias flexibles con los cambios de mercado y de adaptación al medio sobre el que se interactúa. Un ejemplo de esta flexibilidad y adaptación son las tácticas de los bancos electrónicos. En un principio nacieron con la estrategia de ser independientes a su matriz, buscando una nueva cuota de mercado que no podían alcanzar los modelos tradicionales de oficinas y sucursales, ofreciendo paquetes bancarios en otras condiciones, seguimiento de las transacciones por parte del cliente, no existencia de recibos, etc. Pero la estrategia inicial de comercio electrónico planteada no ha funcionado por diferentes razones socioeco-

\section{Enlaces de interés sobre comercio electrónico}

Asociación Española de Comercio Electrónico (Aece) http://www.aece.org

Asociación para la Investigación de los Medios de Comunicación (Aimc)

http://www.aimc.es

Asociación de Usuarios de Internet (Aui)

http://www.aui.es

Internet Society (Isoc)

http://www.isoc.org

Nua

http://www.nua.ie/surveys/

Electronic Commerce Policy

http://www.ecommerce.gov/

Academic Marketing Journals

http://www.tilburguniversity.nl/faculties/few/marketing/links/journal I.html

Web Marketing Info Center

http://www.wilsonweb.com/webmarket/ezines.htm

Advertising Research Resource Center

http://advertising.utexas.edu

Center for eBusiness@MIT

http://ebusiness.mit.edu/resources

Center for Research in Electronic Commerce (Crec) http://cism.bus.utexas.edul

Comercio Electrónico Global

http://www.e-global.es/libros.html

CEN/Isss. Information Society Standardization System http://www.isss-awareness.cenorm.be/ES/inicio_ frame_es.htm

Cordis. IST. Key Action 2. New Methods of Work and Electronic Commerce

http://www.cordis.lu/ist/ka2/welcome.html

CommerceNet en España

http://www.commercenet.org

nómicas y por la propia evolución de internet. Por esta razón se reestructuran de nuevo las políticas, orientándose hacia bancos electrónicos que ofertan el valor añadido que no pueden ofrecer sus "bancos madre".

\section{Definir los objetivos del proyecto.}

Una pequeña relación incluiría los siguientes:

—Captar y fidelizar clientes.

-Abrir y optimizar líneas de negocio para minimizar el tiempo de respuesta. 
-Proporcionar servicios que automaticen las interacciones y/o transacciones con los clientes.

- Consolidar servicios existentes en nuevos canales de comunicación y comercio.

- Ofertar productos y servicios a medida atendiendo a la diversidad del cliente.

-Reingeniería del conocimiento a partir de los diferentes canales implementados en la organización.

-Desarrollar y focalizar canales de imagen organizativa corporativa.

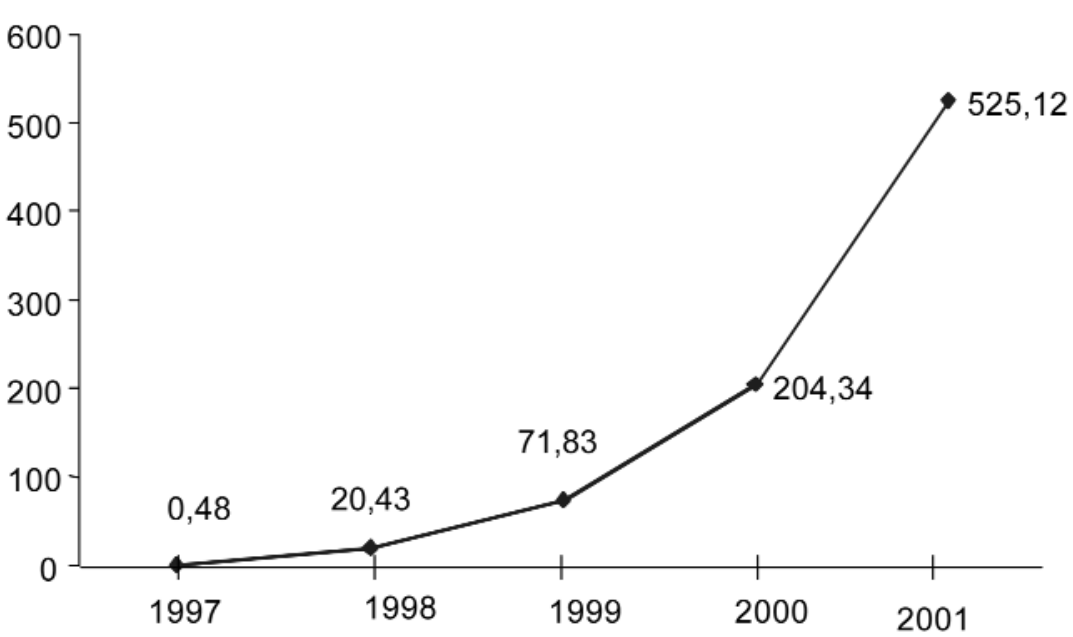

Figura 1. Crecimiento del comercio electrónico frente al consumidor final en España en millones de euros. Fuente: Aece 2002 (Asociación Española de Comercio Electrónico).

- Mejorar la eficacia de las operaciones con un servicio o producto web que coordine los procesos de transacción y negocios de comercio electrónico.

-Aumentar los ingresos o relaciones con canales mediante procesos automatizados.

- Reducir costes a partir de la centralización de información entre distribuidores y compradores.

\section{Definir los requerimientos.}

La estrategia en el diseño de un proyecto de comercio electrónico es diferente para un servicio en funcionamiento que para otro nuevo y diseñado exclu-

\section{Empresas relacionadas con comercio electrónico}

\section{Oracle}

http://www.oracle.com

SAP

http://www.sap.com

Siebel

http://www.siebel.com

Vignette

http://www.vignette.com

Cisco

http://www.cisco.com

Peoplesoft

http://www.peoplesoft.com

\section{Clarify}

http://www.nortelnetworks.com

IBM

http://www.ibm.com sivamente para internet. La virtualización de un servicio existente requiere un estudio previo de viabilidad que indique: si existe una demanda o hay que crearla; cuál es el operativo necesario para integrar los procesos de negocio ya creados; si implementar el servicio requiere nuevos procesos de trabajo; y la capacidad de asumir nuevos desarrollos tecnológicos. En consecuencia, deben enfrentarse a los mismos problemas y retos de las empresas puntocom.

\section{«El éxito en la implementación de un CRM y VRM reside en el cruce del front office y el back office de la organización»}

Los requerimientos necesarios para implementar un proyecto de comercio electrónico deben cumplir la máxima flexibilidad y escalabilidad necesaria para generar productos en todos los canales posibles de comunicación con el cliente, integrados en pasarelas de sistemas de pago o transacciones seguras, con la capacidad de atraer y fidelizar a cada uno de los clientes que entran en el circuito del sistema, y todo ello con una visión sistémica, tal y como se puede observar en la figura 2 .

Esta estrategia transversal de comercio electrónico es apoyada por el conjunto de componentes y herramientas que constituyen el sistema integral de la organización. Por lo tanto, se ha de apoyar en módulos de: información electrónica sobre los clientes, servicios al cliente online, estrategias de marketing, gestión de pagos, gestión de seguridad, logística de productos, integración de aplicaciones internas (back-office).

Estos módulos se encuentran a un mismo nivel en la jerarquía de la empresa y deben estar interrelacionados con los dispositivos front-office y back-office de la 
organización, tales como: su sitio web, la infraestructura organizativa, los procesos de gestión de información y conocimiento, así como el modelo de comercio electrónico que conforman el sistema global de la organización

\section{Planificar, diseñar y desarrollar el sitio web.}

Se debe garantizar que el sistema es capaz de atraer nuevos clientes, atender a los ya registrados, desarrollar nuevos mercados y canales, crear nuevos productos, ofrecer garantía de seguridad, y transparencia en las transacciones. Para ello es necesario emplear herramientas y técnicas de diseño e implementación de sitios web, algunas de las cuales, familiares para los documentalistas, son:

-Visualización de contenidos (mapas web, buscadores internos y externos, faqs, directorios, etc.).

- Técnicas de usabilidad (estructura de la información, distribución de espacios, accesibilidad, etc.).

-Técnicas de marketing electrónico (stickiness ofrecer varias opciones y links para mantener al visitante conectado más tiempo--), optimización de metaetiquetas, posicionamiento web, banners, one to one —marketing personalizado-, etc.).

-Certificaciones de calidad y accesibilidad (validación de W3C, Bobby, etc.).

- Seguridad electrónica (firewalls, codificación de datos, etc.).

- Herramientas de fidelización (VRM, $C R M$, etc.).

- Sistemas de pago (tarjeta, dinero electrónico, etc.).

\section{Fidelización del cliente: VRM y CRM}

Intentaremos dar un sentido lógico a este conjunto de herramientas que se caracterizan por girar en torno a la RM (gestión de la relación con las personas) y presentamos a continuación algunas de las más importantes y utilizadas:

-VRM (visitor/vendor relationship management): gestión de las relaciones con los visitantes anónimos de la web.

-CRM (customer relationship management): gestión de las relaciones con los clientes.

-MRM (marketing relationship management): desarrollo de estrategias globales de marketing basadas
Serviclo de comercio elect rónico

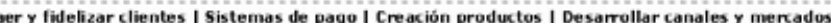

ormación electrónica clientes

ervicios al cliente on-line

ateqias de Marketing

tión de pago

iôn de seguridad

de productos
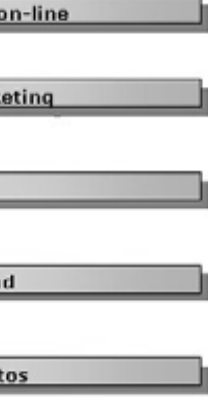
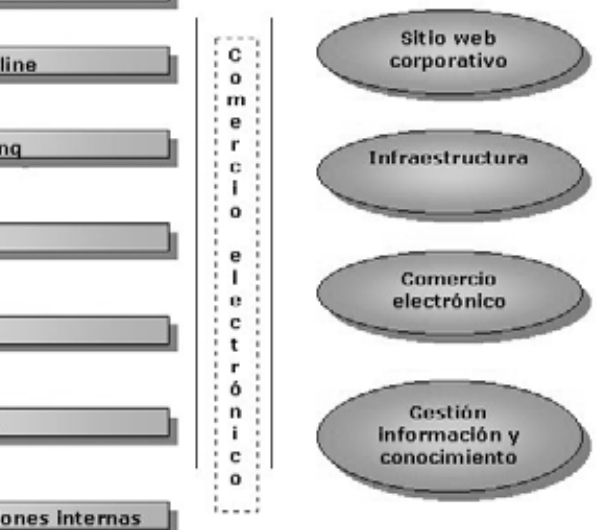

Figura 2. Requerimientos de un proyecto de comercio electrónico

en la gestión de las relaciones con los clientes, a partir de tecnologías que transforman la información que tenemos sobre ellos, ofreciendo campañas más personales y relevantes sobre las necesidades detectadas.

-ERM (employee relationship management): gestión de las relaciones con los empleados. Igual que con los clientes, es básico tener una relación estrecha con los trabajadores, conocerlos y que se sientan a gusto.

-PRM (provider/partner relationship management): gestión de las relaciones con los proveedores. En este caso es importante el trato con el proveedor y establecer las herramientas y canales adecuados de relación con el mismo.

Centrándonos en el CRM y VRM, el objetivo de estas herramientas es permitir gestionar de una forma fácil y acorde con las necesidades de la organización, la ingente información recabada día a día sobre los usuarios registrados y no registrados de nuestra plataforma, procedentes de los diferentes canales de comunicación abiertos.

\section{VRM: usuario anónimo.}

La utilización de estrategias de atracción de clientes potenciales hacia los servicios y productos de un sitio web no es suficiente para fidelizar a un usuario. Además, se convierte en una tarea ardua si no se encuentra registrado ni identificado por el sistema: nos encontramos ante un visitante anónimo y virtual, y como sucede en muchas de las ocasiones, reticente a dar datos personales.

¿Cómo podemos saber lo que atrae de nuestros servicios o productos a un usuario que desconocemos?, ¿cómo ofrecerle un servicio de calidad a medida si no conocemos su perfil de interés? Estas son algunas de 
las preguntas a las que los sistemas $V R M$ intentan dar respuesta, pues a través de los logs, cookies y marcas, tratan y analizan la información volcada en las bases de datos. Posteriormente la cruzan con datos de la organización, permitiendo estudiar el comportamiento del usuario y establecer patrones para modificar los contenidos a partir de las necesidades detectadas.

a. Ficheros log y marcas. Los logs, brevemente, son el registro histórico de todo lo que sucede en un sitio web, con eventos tales como: entrada de usuarios (su dirección ip), lugar de donde provienen, por qué páginas se mueven, fechas, entre otros. Según el servidor, tienen una parametrización por defecto que puede ser susceptible de cambio atendiendo a las necesidades de información para su posterior análisis, aunque la producción de estándares para la recogida de información es cada vez mayor, por ejemplo el W3C (World Wide Web Consortium) trabaja en los atributos básicos de recogida de información de los logs.

http://www.w3.org/TR/WD-logfile

Otro ejemplo es el formato generado por el National Center for Supercomputing Applications denominado «Ncsa combined log format»: client ident user date petition status bytes referer user-agent.

Ejemplo de registro log:

223.10.215.126 [xxx] [yyy] [08/Feb/2002:13:45:29 +0100] «GET /index.html HTTP/1.0» 20042898 «-» «Mozilla/4.0 (compatible; Msie 5.0; Windows 98)»

Estos ficheros históricos presentan una gran complejidad en su tratamiento por el volumen de información que generan, pero hay diferentes herramientas en el mercado que ayudan en su análisis y comprensibilidad. Un ejemplo de ello es el WebTrends Traffic Analy-
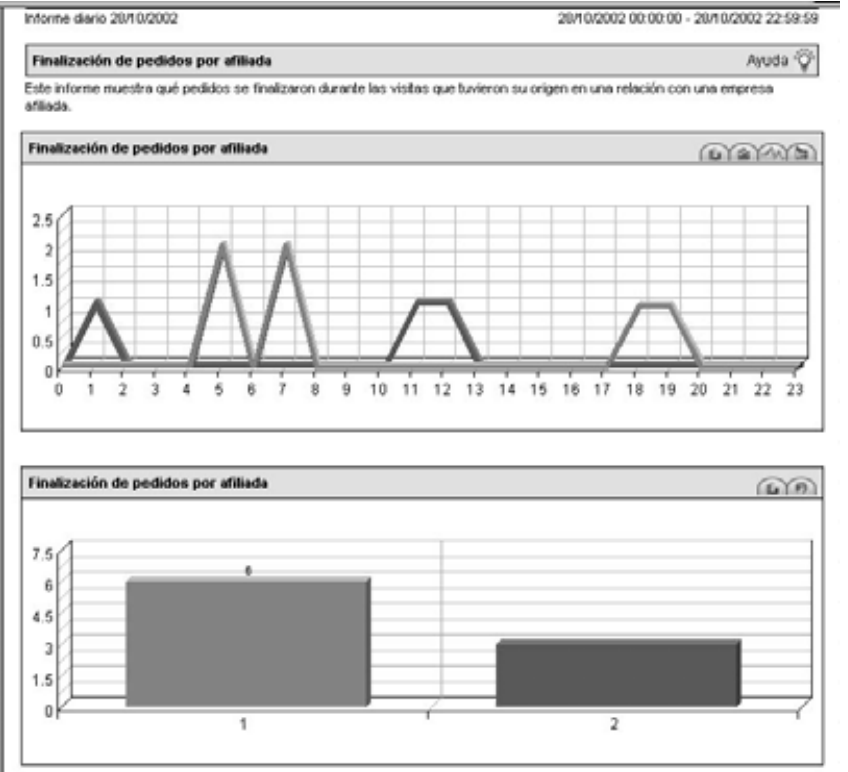

Finaszzeción de pedidos por aflitide sis, que presenta la información en un formato más inteligible (figura 3 ).

A su vez existen programas que a partir de algoritmos más complejos analizan la información de los logs para convertirla en conocimiento estratégico. Estas aplicaciones de web mining elaboran y sintetizan patrones de navegación y uso de los contenidos de los visitantes de un sitio web, segmentan a los usuarios en función de su comportamiento y preferencias, seleccionando los contenidos más adecuados a mostrar en función de su perfil.

\section{«VRM y CRM son herramientas para el desarrollo de relaciones comerciales basadas en la ges- tión personalizada de los clien- tes para obtener una mayor fi- delización y duración de la re- lación»}

Las marcas son utilizadas para establecer un mayor control y una mayor eficiencia en la recogida de información sobre los materiales del sitio web. Con ellas podemos saber si los contenidos tienen el suficiente valor añadido como habíamos imaginado en su creación, aportando datos específicos sobre determinados espacios del sitio web. La ventaja respecto a los logs es que la información que generan es menor y más específica; la desventaja es no poder controlar todos los contenidos y que un error de ubicación puede provocar no descubrir las zonas más demandas por los usuarios.

b. Cookies. Son ficheros de texto instalados en el disco duro de los usuarios a partir de la navegación por webs. Se suelen alojar en la carpeta de ficheros temporales de internet o en una carpeta homónima (caché). Este fichero almacena información sobre el usuario: qué visita, qué lee, qué fotos mira, qué ficheros descarga, etc. Su empleo por parte de algunos colectivos es mirada con recelo, pero lo que intentan es facilitar y adecuar contenidos a las expectativas del navegante. Un ejemplo de utilización es la entrada a una página principal donde nos indican el idioma a escoger. Si tenemos una cookie de este sitio comprobaremos que, en la siguiente visita, el 
sistema nos llevará directamente a los contenidos sin pasar por la elección de idioma.

\section{CRM: usuario registrado.}

$C R M$ es definido como "toda estrategia empresarial que implica un cambio de modelo de negocio centrado en la gestión automatizada de todos los puntos de contacto con el cliente, cuyo objetivo es captar, fidelizar y rentabilizar al cliente ofreciéndole una misma cara mediante el análisis centralizado de sus datos"3. Gartner Group lo define como una estrategia empresarial diseñada y centrada en el cliente para optimizar la rentabilidad, los ingresos y la satisfacción de éste.

\section{«El término comercio electróni- co ha obtenido últimamente una mayor divulgación, aunque ha existido bajo diversas for- mas desde hace más de 20 años»}

No existe una definición única, no por falta de acuerdo entre los distintos expertos sino por la velocidad de transformación que tienen estas herramientas y modelos en las organizaciones, pero sí podemos establecer unos conceptos clave a la hora de definirlo:

—Estrategia: más que un conjunto de herramientas es un modelo de organización cuyos procesos se basan en parámetros de, para y por el cliente.

-Atraer, identificar y fidelizar: el objetivo del $C R M$ es atraer clientes a partir de la información generada e identificar patrones de necesidades con el fin de fidelizar a través de una atención personalizada basada en estrategias de marketing one to one, entre otras.

—Gestión de información: su base es la gestión de información que genera el cliente, junto a los datos de la organización a partir de una explotación cruzada con la aplicación de diferentes técnicas de análisis.

No debemos omitir en este conjunto de conceptos clave el beneficio económico de la compañía.

A partir de estas definiciones podemos establecer cuáles son las principales claves de la filosofía $C R M^{4}$, que se pueden sintetizar en:

-Focalización de la gestión de las relaciones: tratar la información del usuario de forma unitaria, recogiendo los datos existentes en los diferentes sistemas.

- Conocimiento de la persona: explotar y analizar los datos sobre los usuarios y rentabilizar el conocimiento obtenido de ellos, para evaluar el nivel de captación y fidelización.

-Integrar múltiples canales: componer los diferentes canales de conexión para las relaciones (contactos vía formularios, llamadas telefónicas, correos electrónicos y/o un documento postal).

-Enfocarse en la satisfacción del cliente: conocer el grado de satisfacción y optimizarlo para mantenerlos y fidelizarlos.

- Integración de todos los sistemas de la organización: integrar para compartir los datos generados por diferentes aplicaciones.

-Individualización y flujos de conocimiento: establecer relaciones y patrones de conocimiento entre servicios/productos y clientes de forma individualizada.

\section{CRM analítico, operacional y cooperativo.}

La función del $C R M$ analítico es identificar y diferenciar al usuario: cuántos son clientes potenciales, cuáles deben recibir una información personalizada, quiénes son rentables a nuestra organización, cómo segmentarlos según el catálogo de productos y servicios, etc.

Por otro lado nos encontramos con el CRM operacional, donde se integran los centros de atención a clientes (call-centers), sitios de comercio electrónico y sistemas automatizados de pagos. Es decir, integra todos los canales de comunicación con el cliente front-

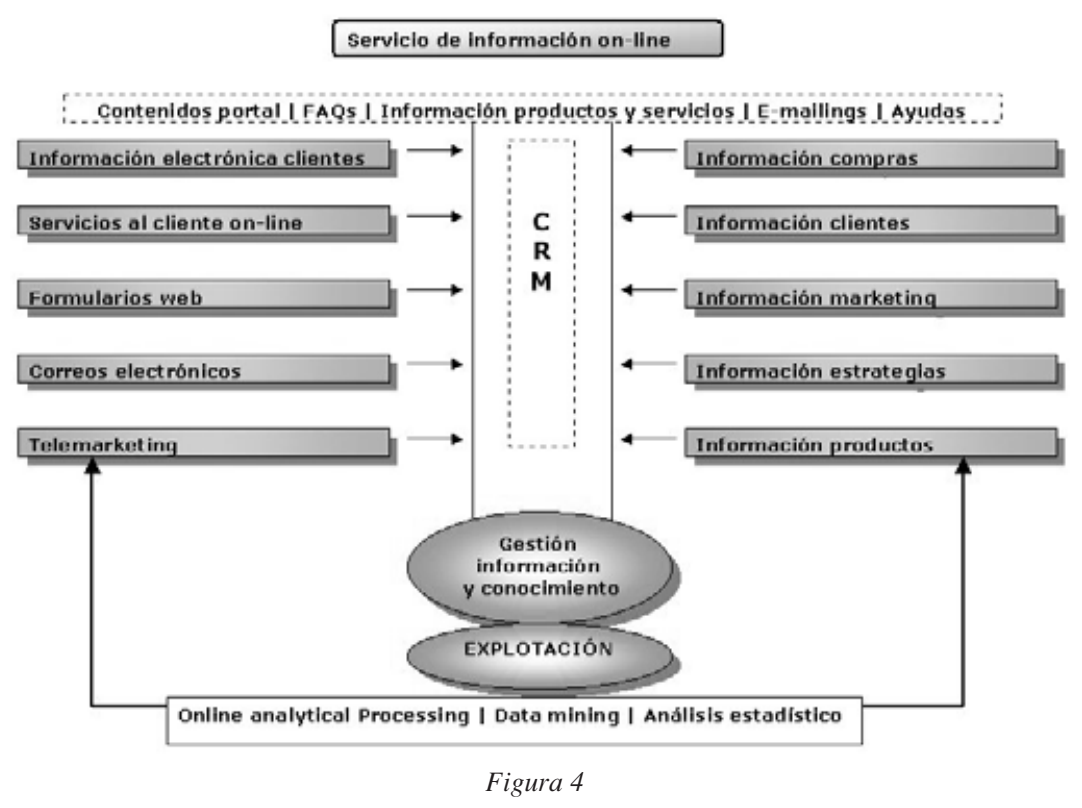


office) con los sistemas de información interna de la organización (back-office).

El $C R M$ cooperativo es el encargado de ayudar en la toma de decisiones y de distribuir la información a partir de entornos colaborativos entre clientes y la organización.

\section{La función del servicio de información en la fidelización del cliente}

Debe controlar cada uno de los procesos de la organización donde está involucrado. Por ello, debe gestionar toda la información relativa a la empresa y su relación con el cliente: contenidos del site, faqs (preguntas más frecuentes), servicios de atención al cliente, información de los productos y servicios, campañas de marketing, ayudas online, entre otros (figura 4).

Para gestionar correctamente el servicio, alcanzar una estrategia de marketing one to one y la máxima personalización posible, es necesario trabajar directamente con:

-información electrónica que generan los clientes,

— servicios de información para el cliente,

- gestionar y organizar los contenidos de los formularios de recogida de información,

- organizar la información de los correos electrónicos generados por los clientes, y

- canalizar la información generada desde las campañas de marketing.

El conjunto de la información, una vez recopilada en el $C R M$ y/o $V R M$, debe ser estructurado mediante técnicas de gestión de información y conocimiento para su posterior explotación. El análisis puede ser a partir de técnicas de olap (online analytical processing, o análisis de empresas basados en modelos de datos multidimensionales), data mining o análisis estadísticos.

Algunos de las resultados que podremos obtener son: número de accesos que recibimos, qué contenidos son los más vistos, visitas a partir de las campañas de captación y de dónde vienen, cómo nos encuentran, qué palabras clave utilizan para localizarnos, cuál es el perfil de quien utiliza nuestro servicio, quién de nuestros usuarios utiliza más el servicio, cuál es el perfil de quienes realizan la compra, reclamaciones, demandas más solicitadas, tiempo de resolución de las demandas, volumen de ventas, etc. La información es analizada y ha de producir un feed-back útil para los componentes del sistema con el que se podrán realizar las actuaciones oportunas con el fin de obtener la mayor personalización posible y satisfacer todas las necesidades que los clientes han demandado o han sido detectadas por el sistema de información.

\section{Mecanismos de pago electrónico y seguridad}

Actualmente existe una amplia diversidad de formas para realizar el pago electrónico. La interoperabilidad entre ellas sería deseable, aunque en algunos casos este requisito puede introducir un sobrecoste apreciable en las transacciones. Tanto la Unión Europea como los EUA favorecen acuerdos de la industria como mejor forma de incrementar la interoperabilidad, aunque sin descartar la necesidad de introducir normas generales.

En general, los vendedores a través de internet tratarán de soportar el mayor número posible de sistemas de pago con el fin de atraer más clientes (como ocurre ahora en el comercio tradicional). La iniciativa Jepi (Joint electronic payment initiative) del Consorcio $W 3 C$ y la asociación CommerceNet define un protocolo que permite negociar automáticamente cuál de los múltiples métodos de pago aceptables para el comprador y el vendedor se debe utilizar en una transacción particular.

\section{Pagos con tarjeta de crédito a través de in- ternet.}

Un ejemplo de método de pago electrónico es el protocolo SET (secure electronic transaction) definido por MasterCard y Visa con la colaboración de otras importantes compañías como IBM, Microsoft y Netscape, y que permite hacer transacciones seguras con tarjeta de crédito a través de internet. Otro método empleado es el SSL (secure socket layer), que encripta la información de formularios web hasta el servidor de comercio electrónico

En el caso de España, la Agencia de Certificación Electrónica, en la que participan Telefónica, Ceca, Sermepa y Sistema $4 B$, emite ya certificados SET. Para los usuarios estos certificados se ofrecerán como un servicio más de los bancos y cajas, con la consiguiente imagen de seguridad dada por estas entidades y, en principio, con buenas perspectivas de aceptación por los consumidores. Otros sistemas de pago electrónico basados en tarjetas son CyberCash y First Virtual.

Un modelo simple está compuesto por:

-Comprador: accede a la web del servicio a través de internet, selecciona el producto, realiza una pasarela al TPV (acceso al servidor seguro del banco de forma transparente al usuario), introduce el número de la tarjeta, el banco devuelve una información de aceptación de la compra por correo electrónico, un link a 
una web o acceso a una parte del servidor donde el usuario tiene su cuenta.

-Vendedor: es la empresa con el servicio. Puede utilizar dos procedimientos para realizar los cobros: un acceso directo al TPV del banco para compras sencillas o, si el sistema es más complejo, tiene un programa residente con conexión al TPV. Tras la compra el vendedor entra en los informes de las compras que, dependiendo del banco, serán más o menos completos.

-Bancos. Obtienen dos beneficios: por un lado se encuentra el del vendedor, que actualmente debe abonar entre un $3 \%$ a un $5 \%$ de la transacción, y por otro lado el del comprador, que utiliza su tarjeta para realizar la compra.

Algunos ejemplos en España son:

-Banco Popular.

http://www.bancopopular.es/productos/ProdPlantilla. asp?Prod $=108$

\section{-Banco Atlántico.}

http://www.batlantico.es/batlantico/tpvv_web/pres.ht $m l$

-Banco Santander Central Hispano.

http://www.bsch.es/es/grupo/financieraleuro/todo/age ntes/medios pago.jsp

-Banco Sabadell.

http://www.bsshopping.com/wl-docpub/user/cas/infocomer.jsp

- La Caixa.

http://comercios.lacaixa.es/Canales/Contenido/0,105 9,1-42-676-1,00.html? loc $=$ P003-2-1-42-549-23572083-2-3698-3

\section{- Caja Rural.}

http://shop.cajarural.com:8080/cgibin/ncommerce3/ExecMacro/ruraltienda/comercios_a sociados.d $2 w /$ report

\section{Cheques y órdenes de pago electrónicas.}

En el caso del comercio entre empresas, el pago mediante tarjeta de crédito es mucho menos usual, por lo que un sistema como SET parece claramente menos adecuado que en el escenario anterior. En estos casos, en particular en las pymes, es frecuente el pago mediante cheques. Los sistemas basados en cheques electrónicos pueden reducir considerablemente el coste de procesar los cheques y minimizar el fraude (firma digital en lugar de firma tradicional).

Un ejemplo de sistema de cheque electrónico es eCheck definido por el Fstc (Financial Service Tech- nology Consortium), un consorcio de más de 90 miembros, principalmente bancos, que colaboran de forma no competitiva en el desarrollo de proyectos técnicos. El sistema Fstc utiliza una tarjeta inteligente para implementar un «talonario de cheques electrónicos» seguro. La tesorería estadounidense firmó en junio de 1998 su primer cheque electrónico usando este método, marcando el inicio de un periodo de pruebas del sistema antes de su comercialización.

http://www.echeck.org/

\section{Dinero electrónico.}

Los sistemas de pago citados anteriormente sirven para realizar transacciones electrónicas (cargo en una tarjeta de crédito, orden de pago) sobre dinero no electrónico. Existe otro grupo de sistemas en los que se maneja directamente dinero electrónico, por ejemplo almacenado en una tarjeta inteligente que hace de monedero electrónico. Estos sistemas se basan en el prepago, es decir la conversión previa de dinero real en electrónico. La información actual es que estos sistemas han sido un fracaso, tomando como ejemplo $C y$ berCash, DigiCash, Millicent...

\section{Seguridad en las transacciones y codificaciones online}

Para el éxito del comercio electrónico es necesario asegurar la confianza del consumidor en la fiabilidad y protección de las transacciones comerciales. De hecho, los clientes son reacios a proporcionar información sobre sus tarjetas de crédito en internet. Los tipos de operaciones dependen de la naturaleza de los datos (o contenidos) que se vayan a enviar por la Red. En concreto se diferencia entre datos públicos, con copyright, confidenciales y secretos.

Los requerimientos para la seguridad de las transacciones $^{5}$ son básicamente 3 :

-Privacidad: debe ser conservada junto con la inviolabilidad, de forma que los intrusos no puedan entender el contenido del mensaje.

-Confidencialidad: el rastro de las operaciones no puede ser accesible desde la red pública y los intermediarios no pueden realizar copias de las mismas a menos que estén autorizados para ello.

-Integridad: no pueden ser saboteadas o interferidas.

Para asegurar tales exigencias se está implementando un conjunto de sistemas de codificación o encriptación de la información:

-Codificación con clave privada: también denominada simétrica, implica el uso de una clave compar- 


\section{Complete su colección de IWE/EPI}

Deseo recibir las siguientes publicaciones:

$\square$ ejemplares de la Bibliografía IWE/EPI I992-200 I por correo postal impresos en papel (gratis).

la Bibliografía IWE/EPI 1992-200I por correo electrónico en formato PDF (gratis).

$\square$ álbumes de cd-rom con el texto íntegro de los primeros 100 números de la revista a 30 euros cada disco (uno es gratis para los suscriptores).

$\square$ _ ejemplares de los siguientes números atrasados de la revista (por favor, envíenme presupuesto).

Nombre:

Institución:

Dpto.: ...............................................................NIF institucional:

Dirección:

Código postal:

Ciudad:

País:

Teléfono:

Fax:

Correo-e:

Envíe este boletín por correo postal, fax o correo electrónico, a esta dirección:

Swets Blackwell

Caspe, 46

08010 Barcelona

España

Tel.: +34-932 70। 144; fax: 93270 I I45

mnzang@es.swetsblackwell.com

tida para la encriptación del transmisor y la decodificación del receptor.

-Codificación con clave pública: es conocida como codificación asimétrica e implica el uso de dos claves: una para codificar el mensaje u otra diferente para decodificarlo.

-Firmas digitales: mecanismo criptográfico que realiza una función similar a una escrita. Se utiliza para verificar el origen y contenido de los mensajes. Por ejemplo en un e-mail se puede comprobar quién lo firmó y que los datos no fueron modificados después de ser firmados.

-Certificados digitales: proporcionan una vía adecuada para que los participantes en una transacción de comercio electrónico puedan confiar cada uno en el otro mediante el uso de una tercera parte común.

Las firmas digitales pueden establecer la identidad de las partes en un entorno de red, así como asegurar la integridad de los datos. A pesar de los avances tecnológicos en este ámbito, los complicados enfoques sobre la validez legal de las firmas digitales entorpece su utilización. Las firmas, digitales o no, tienen 3 funciones legales:

- La firma prueba que el documento es atribuible al signatario.

- El acto de firmar llama la atención del signatario sobre su significado legal.

-El acto de firmar expresa la aprobación o autorización del documento.

En España - a través del Real Decreto-ley 14/1999 de 17 de septiembre- se entiende como firma electrónica al conjunto de datos, en forma electrónica, anejos a otros datos electrónicos o asociados funcionalmente con ellos, utilizados como medio para identificar formalmente al autor o a los autores del documento que la recoge. Se introduce una variante de firma electrónica denominada avanzada que se define como aquella que permite la identificación del signatario y ha sido creada por medios que éste mantiene bajo su exclusivo control, de manera que está vincula- 
da únicamente al mismo y a los datos a los que se refiere, lo que hace posible que sea detectable cualquier modificación ulterior de éstos.

La firma electrónica avanzada, siempre que esté basada en un certificado reconocido y producida por un dispositivo seguro de creación de firma, tendrá el mismo valor jurídico que la manuscrita, y será admitida como prueba en juicio. Para ello debe tener un certificado reconocido donde se pueda comprobar que ha sido expedido por un prestador de servicios de certificación acreditado y el dispositivo seguro de creación de firma con el que ésta se produzca se encuentre certificado, con arreglo a lo establecido en el artículo 21.

Pero se ha de tener en cuenta que la firma electrónica que no reúna todos los requisitos previstos en el apartado anterior no se le negarán efectos jurídicos ni será excluida como prueba en juicio por el mero hecho de presentarse en forma electrónica.

\section{Conclusiones}

- Los proyectos de comercio electrónico deben ir acompañados de una buena estrategia de negocio junto a una buena estrategia técnica, con la suficiente flexibilidad para adaptarse a los continuos cambios del mercado electrónico.

—El comercio electrónico no está constituido únicamente por un conjunto de herramientas tecnológicas para atender las ventas de servicios y/o productos: es un modelo de organización que abarca cada uno de los procesos de la empresa.

-El comercio electrónico debe integrarse en el sistema de información de la organización dando lugar a un proceso continuo de feed-back útil para la toma de decisiones en la organización.

-El desarrollo de modelos de comercio electrónico ha de integrar los front-office y back-office de la organización.

-Algunos de los elementos claves en su implantación son la integración de la información y conocimiento del cliente con el sistema de información de la organización.

- La estrategia de comercio electrónico basada en el cliente debe contar con herramientas como $C R M$ y $V R M$ para aplicar técnicas one to one.

\section{Notas}

1. What is electronic commerce? Consultado en: 18-09-02.

http://www.martech-intl.com/best2/intro.htm

2. COM (97). Iniciativa europea de comercio electrónico. Consultado en: 22-09-02.

http://europa.eu.int/scadplus/leg/es/lvb/l32101.htm
3. García Varcárcel, Ignacio. CRM: gestión de la relación con los clientes. Madrid: Fundación Confemetal, 2001, pp. 45-50. Isbn 84-95428-39-3.

4. Berbel, Genís; Capillas, Ramón. Gestión de la relación con las personas en la web: VRM y CRM, 2002. Consultado en: 18-09-02.

http://www.uoc.edu/web/esp/art/uoc/berbel_capillas0302/berbel_capillas0 302.html

5. Kalakota, R.; Whinston, A. B. Electronic commerce: a manager's guide. Massachusetts: Addison-Wesley, 1997, pp. 125-137.

\section{Bibliografía}

Archer, Norm; Yuan, Yufei. "Managing business to business relationships throughout the e-commerce procurement life cycle". En: Internet research: electronic networking applications and politics, 2001, v. 10, n. 5, pp. 385-395.

Asociación Española de Comercio Electrónico. Consultado en: 25-09-02. http://www.aece.org/docs/resumenB2C2002.pdf

Bergeron, Bryan P. Essentials of CRM: customer relationship management for executives. 2002. Isbn 0-471-20603-2.

Comisión del Mercado de las Telecomunicaciones. Consultado en: 01-10-02. http://www.cmt.es/cmt/centro_info/publicaciones/pdf/comercio-e/2T02.pdf

Curry, Jan. CRM, cómo implementar y beneficiarse de la gestión de las relaciones con los clientes (customer relationship management). Barcelona: Gestión 2000, 2002. Isbn 84-8088-723-0.

Damanpour, Faramarz. "E-business e-commerce evolution: perspective and strategy". En: Managerial finance, 2001, v. 27, n. 7, pp. 16-32.

García Mas, Francisco Javier. Comercio y firma electrónicos: análisis jurídico de los servicios de la sociedad de la información. Valladolid: Lex-Nova, 2002.

Gómez Cáceres, Diego; Corbalán Sánchez de las Matas, Luis. Mercados electrónicos: nuevos sistemas de pago. Madrid: Esic, 2001.

Laudon, Kenneth C.; Guercio Traver, Carol. E-commerce: business, technology, society. Boston; Madrid [etc.]: Addison Wesley, 2002.

Martínez Nadal, Apolonia. Comercio electrónico, firma digital y autoridades de certificación. Madrid: Civitas, 2001. Isbn 84-470-1651-X.

Martínez Usero, José Ángel; Lara Navarra, Pablo. “Aspectos clave en la creación de un sitio web para utilidades de comercio electrónico: posibles aplicaciones en los proyectos de biblioteca digital". En: XI jornadas bibliotecarias andaluzas, 2000.

Motin, Jean-Henry. "Commercialization of electronic information". En: Ieee multimedia systems'99, 1999.

Onmonbekov, T.; Bello, Daniel C.; Gilliland, David I. “Adoption of electronic commerce tools in business procurement: enhanced buying center and processes". En: Journal of business \& industrial marketing, 2002, v. 17, n. 2/3, pp. 151-166.

Sala i Andrés, Anna Maria. «La autoría en las manifestaciones electrónicas». En: La ley, 2000, septiembre, n. 5.140, pp. 1-4.

Siebel, Thomas M. Principios del e-business. Barcelona [etc.]: Granica, 2001. Isbn 84-7577-897-6.

Wen, Joseph; Chen, Houn-Gee; Hwang, Hsin-Ginn. "E-commerce web site design: strategies and models". En: Information management \& computer security, 2001, v. 9, pp. 5-12.

Zingale, Alfredo. New economy emotion: engaging customer passion with e-CRM. Chichester: John Wiley \& Sons, 2001. Isbn 0-470-84135-4.

Pablo Lara Navarra, profesor coordinador del área de gestión de la información y conocimiento de la UOC. plara@uoc.edu

José Ángel Martínez Usero, consultor de la licenciatura en documentación de la UOC y profesor asociado de la Universidad Carlos III.

jmartinezu@uoc.edu 